\title{
UTILIDAD DEL MENOPAUSE RATING SCALE (MRS) EN INDÍGENAS COLOMBIANAS EN CLIMATERIO
}

\section{VALIDITY AND RELIABILITY OF MENOPAUSE RATING SCALE IN COLOMBIAN INDIGENOUS POPULATION}

\section{TÍTULO CORTO: UTILIDAD DE MENOPAUSE RATING SCALE (MRS) EN INDÍGENAS}

\section{Álvaro Monterrosa-Castro ${ }^{1}$, Ángel José Paternina-Caicedo ${ }^{2}$, Edwin Herazo-Acevedo ${ }^{3}$, Heidi Celina Oviedo-Acevedo ${ }^{4}$, Adalberto Campo-Arias ${ }^{5}$}

Recibido en agosto 24 de 2015

Aceptado en febrero 05 de 2016

\section{RESUMEN}

La Menopause Rating Scale (MRS) se compone teóricamente de tres dimensiones que evalúan síntomas somáticos, emocionales y urogenitales relacionados con la menopausia. La utilidad de las escalas varía según las características poblacionales y no se cuenta con investigaciones que corroboren estas dimensiones en población indígena. El objetivo fue evaluar la utilidad de las dimensiones y confiabilidad de MRS en indígenas colombianas. Se realizó análisis del patrón de respuesta de MRS en 914 mujeres indígenas, 507 posmenopáusicas y 407 premenopáusicas, entre 40-60 años, media 50,3 años ( $D E=5,9)$. Se estimó alfa de Cronbach para las dimensiones originales y para las que emergieron en el análisis factorial mediante el método de máxima verosimilitud y rotación oblicua promax. MRS mostró alfa de Cronbach: 0,86; la dimensión somática 0,63, la psicológica 0,75 y la urogenital 0,84. La puntuación fue significativamente superior en posmenopáusicas que premenopausia $14,4(\mathrm{DE}=6,4)$ vs. 8,4 $(\mathrm{DE}=5,9) \mathrm{p}<0,001$. El análisis de factores identificó dos factores; el primero que dio cuenta del 39,9\% de la varianza (ítem 1,7,8,9,10,11) y el segundo del 14,2\% (ítem 2,3,4,5,6). La primera dimensión tuvo alfa de Cronbach 0,86; y la segunda 0,81. MRS presentó alta consistencia interna, adecuada validez nomológica y dos dimensiones. Es necesario corroborar el desempeño de los instrumentos en diferentes poblaciones.

Palabras clave: Menopausia; Salud de Poblaciones Indígenas; Climaterio; Calidad de vida

\section{ABSTRACT}

The Menopause Rating Scale (MRS) measures quality of life in menopausal women. It compounds of three dimensions that assess somatic, psychological and urogenital menopausal-related symptoms. However, the validity of the scales may vary according to population characteristics, and there are no validations to date of MRS in American indigenous

1. MD. Grupo de investigación "Salud de la Mujer”, Facultad de Medicina. Universidad de Cartagena, Colombia. Correo: alvaromonterrosa@gmail.com;

2. MD, M.Sc. Grupo de investigación “Salud de la Mujer”, Facultad de Medicina. Universidad de Cartagena, Colombia. Correo: angel.paternina@gmail.com

3. MD, M.Sc, PhD (c). Human Behavioral Research Institute. Bogotá, Colombia. Correo: eh@comportamientohumano.org

4. MD, M.Sc. Human Behavioral Research Institute. Bogotá, Colombia. Correo: hoviedo@unab.edu.co

5. MD, M.Sc. Universidad del Magdalena. Santa Marta, Colombia. Correo: acampoa@universidadmag.onmicrosoft.com 
population. To assess the validity of MRS in Indigenous Colombian women during menopause. A research was done a sample of 914 indigenous women, 507 postmenopausal women and 407 premenopausal. They were between 4049 years-old, with a mean age of $59.3 \pm 5.9$ years. MRS was applied to all enrolled women. Cronbach's alpha was applied for the original proposed dimensions, and the dimensions from the results of factor analysis and maximum likelihood methods. A Promax rotation was applied to analysis. MRS showed a Cronbach's alpha: 0.86 . The somatic dimension: 0.63 , the psychological dimension: 0.75 , and urogenital: 0.84 . Score was greater in postmenopausal compared to premenopausal, $14.4( \pm \mathrm{SD}, 6.4)$ versus $8.4( \pm \mathrm{SD}, 5.9)(\mathrm{P}<0.001)$. The factor analysis showed two dimensions. The first dimension included items 1,7,8,9,10,11; and accounted for $39.9 \%$ of variance. The second dimension included items $2,3,4,5,6$; explaining $14.2 \%$ of variance. Cronbach's alpha was 0.86 for the first dimension and 0.81 for the second dimension. MRS showed high internal consistency and adequate nomological validity. The factor analysis resulted in two dimensions. These results evidence the need to better assess the validity of the instruments in different populations.

Keywords: Menopause; Health of Indigenous Peoples; Climacteric; Quality of life

\section{INTRODUCCIÓN}

$\mathrm{L}^{\mathrm{a}}$ a construcción de instrumentos de medición en salud sigue un proceso complejo ${ }^{1}$. Con base en un concepto clínico, enfermedad o dolencia, se presenta una propuesta de escala de identificación de posibles casos o de evaluación de la severidad de los síntomas².

Estas escalas o instrumentos de medición necesitan mostrar validez y confiabilidad ${ }^{3}$. La validez se refiere a si la escala mide lo que realmente intenta medir, identifica correctamente los casos y la confiabilidad a si la aplicación de la escala en situaciones similares recoge el mismo patrón de respuesta ${ }^{4}$.

Existen varias formas de conocer la validez de una escala. La más sencilla, pero más costosa y laboriosa, es comparar el desempeño de la escala frente al mejor criterio de referencia (Gold standard), es decir, los criterios diagnósticos que se tienen generalmente a partir de expertos clínicos ${ }^{5}$. Otra manera de explorar la validez de una escala y, en consecuencia, de los supuestos teóricos subyacentes, se puede hacer en forma indirecta mediante técnicas estadísticas ${ }^{6}$.

Tradicionalmente, la validez de constructo se ha realizado mediante el uso de la técnica del análisis de factores ${ }^{7}$. Esta alternativa permite conocer o corroborar la agrupación en dimensiones, dominios o factores de un conjunto de ítem, reactivos, incisos, preguntas o síntomas que indagan 0 hacen parte de una condición o enfermedad ${ }^{8}$.

Asimismo, existe otra forma de validez se conoce como validez nomológica, por hipótesis o teórica; esta consiste en comparar las puntuaciones de la escala en distintas poblaciones que en teoría deben mostrar patrones de respuesta distintos, por ejemplo, con Menopause Rating Scale (MRS) se esperaría encontrar puntuaciones mayores en mujeres posmenopaúsicas que en aquellas en el período previo".

Por su parte, la confiabilidad de una escala se puede estimar con una aplicación del instrumento y el cálculo de la consistencia interna, la prueba más conocida es el coeficiente de alfa de Cronbach ${ }^{10}$. O mediante aplicaciones sucesivas para saber la estabilidad de la escala o la sensibilidad al cambio ${ }^{11}$. Para ello se utilizan los coeficientes de correlación de Pearson ${ }^{12}$, Spearman ${ }^{13}$ 0 intraclase $\mathrm{i}^{14}$.

La escala para calidad de vida en la menopausia (Menopause Rating Scale, MRS) consta de dos versiones una inicial de diez ítems (MRS I) ${ }^{15} \mathrm{y}$ una modificada de once ítems (MRS II) ${ }^{16}$. La versión más usada en la última década es la MRS II, que sustituyó por completo la aplicación de la MRS I y, en consecuencia, suele llamarse a la MRS II simplemente MRS. Un análisis de factores inicial informó que la MSR se compone de tres dimensiones, 
cuatro ítems que evalúan síntomas somáticos; cuatro, manifestaciones emocionales o psicológicas; y tres, molestias urogenitales ${ }^{16}$.

Del mismo modo, estudios disponibles en diferentes poblaciones alrededor del mundo informan que la MRS es una escala confiable con valores adecuados del coeficiente de alfa de Cronbach, alrededor de $0,80^{17,18}$. Sin embargo, la consistencia interna de cada dimensión varía mucho más, entre 0,60 y $0,87^{17}$.

Es bien conocido en psicometría o clinicometría que el desempeño de las escalas de medición cambia en forma importante según las características de la población. De tal suerte que las dimensiones y la consistencia de los instrumentos varían en los distintos contextos y colectivos $^{19}$. Esto hace necesario antes de la aplicación de un instrumento en una población distinta a la que participó en el proceso de diseño se corrobore el desempeño del mismo y la equivalencia lingüística de cada ítem ${ }^{20}$. La mayoría de los estudios con el uso de la MRS se han limitado a presentar la frecuencia de síntomas en mujeres de los cinco continentes no indígenas, afrodescendientes, caucásicas, hispanas asiáticas, e indígenas ${ }^{21-32}$; sin explorar o corroborar la estructura de dimensiones que se propuso inicialmente y explorar explícitamente la validez nomológica. Además, las diferencias en el patrón de síntomas y de respuestas en mujeres de diferentes contextos sociales y culturales sugieren un desempeño factorial similar ${ }^{19}$. A la fecha no se cuenta con investigaciones que informen las dimensiones, la validez nomológica formalmente y la consistencia interna de la MRS en población indígena.

El objetivo de este análisis fue evaluar la utilidad de las dimensiones y la confiabilidad de la escala MRS en mujeres indígenas colombianas en Climaterio.

\section{MATERIALES Y MÉTODOS}

Se realizó un análisis cuantitativo del patrón de respuesta de la MRS en 914 mujeres indígenas, 407 en premenopausia y 507 posmenopáusicas. Las edades del grupo de participantes se encontraban entre 40 y 59 años, media 50,3 años $(\mathrm{DE}=5,9)$. Mujeres indígenas colombianas naturales y residentes de tres asentamientos, en departamentos con presencia de nativos autóctonos. Participaron mujeres de las etnias wayuú, asentada en La Guajira, en el norte del país; Zenú, residente en resguardos del departamento de Córdoba; y nativas de la Amazonía, al sur de Colombia.
La MRS es una escala auto-administrada para cuantificar calidad de vida en la menopausia. Es un instrumento de origen alemán, con traducciones a varios idiomas. Se compone de once ítems divididos en tres sub-escalas o dimensiones: (A) somática-vegetativa: sofocos, molestias cardiacas, trastornos del sueño, molestias musculares y de las articulaciones [ítem 1, 2, 3, 11, respectivamente]; (B) psicológica: estado de ánimo deprimido, irritabilidad, ansiedad, cansancio físico y mental [ítem 4, 5, 6, 7, respectivamente]; y (C) urogenital: problemas sexuales, de vejiga y sequedad de la vagina [ítem 8, 9, 10, respectivamente]. Estos ítems se califican como: 0 [ausente], 1 [leve], 2 [moderado], 3 [severo] y 4 [muy severo] $]^{15,16}$.

Profesionales de la salud o trabajadoras sociales y pertenecientes, con actividad laboral y/o residentes en las mismas comunidades, realizaron la traducción de la MRS del español a los dialectos de las diferentes etnias. Cuando las mujeres hablaban español o simultáneamente español y dialecto, la escala se entregó en español y fue auto-aplicada, en concordancia con los criterios de los proponentes originales del formulario. Cuando se necesitó realizarla en alguno de los dialectos, la encuestadora hizo la traducción simultánea leyendo los apartes del formulario.

Se consideraron dos grupos. Premenopáusicas, todas las mujeres que presentaban las siguientes condiciones: [A] ciclos menstruales regulares (variaciones menores a siete días). [B] Ciclos menstruales irregulares (variaciones superiores a siete días). [C] Las que al momento de ser incluidas tenían amenorrea inferior a doce meses. Por tanto, es un grupo que involucra tanto las verdaderamente premenopáusicas como perimenopáusicas. El otro grupo son las posmenopáusica, o se las que tenían uno o más años sin presentar sangrados menstruales.

Para conocer la confiabilidad de la escala se estimó el coeficiente de alfa de Cronbach $^{10}$. Asimismo, se calculó la correlación de Pearson corregida de cada ítem con la puntuación total, es decir, la correlación del ítem con la suma de la puntuación en los ítems restantes de la escala y el alfa de Cronbach si el ítem se excluyera de la escala ${ }^{10}$.

Para explorar la validez nomológica se compararon la media y la desviación estándar de las puntuaciones en mujeres en la posmenopausia frente a las mujeres en la premenopausia mediante la prueba de t de Student.

Para iniciar el análisis de factores y conocer si los ítems se agrupaban en diferentes dimensiones de un constructo 
subyacente se calculó el coeficiente de Kayser-MeierOlkin (KMO) ${ }^{33}$ y la prueba de esfericidad de la muestra de Bartlett $^{34}$. Se esperó para la prueba KMO un valor mayor de 0,800 y una prueba de Bartlett con un valor de probabilidad menor de $0,05^{35}$.

El análisis de factores se llevó a cabo bajo el método de máxima verosimilitud. Dado que se considera que los ítems que hacen parte de la MRS se agrupan en varios factores altamente correlacionados entre ellos se llevó una rotación oblicua promax para mayor claridad de la solución factorial ${ }^{8}$. Se consideró que un ítem pesó en un factor si mostró valor de coeficiente de 0.550 y que un factor era importante si alcanzó un valor propio superior a 1,40 y se componía por lo menos por tres ítems con coeficientes superiores a 0,550.

\section{Declaración de aspectos éticos}

Estudio que hace parte del proyecto de investigación CAVIMEC (Calidad de Vida en la Menopausia y Etnias Colombianas), inscrito y evaluado por el comité de ética de la Universidad de Cartagena, Colombia. Se tuvieron en cuenta las normas científicas, técnicas y administrativas para investigación en salud, establecidas por la Resolución
008438 de 1993 del Ministerio de Salud de la República de Colombia, y se considera (de acuerdo con el artículo 11, numeral a), al presente estudio como investigación sin riesgos para la población estudiada.

Las mujeres invitadas a participar podían negarse, suspender el diligenciamiento del formulario y dejarlo incompleto si lo consideraban pertinente. Se solicitó el consentimiento informado previo a la aplicación de la encuesta, de acuerdo a la declaración de Helsinski. Participación anónima de las mujeres involucradas. Las encuestadoras, integrantes de las comunidades a encuestar solicitaron las autorizaciones respectivas, de ser pertinente, de las autoridades locales y de los agentes comunitarios o familiares, respetando creencias y comportamiento particulares según los casos.

\section{RESULTADOS}

La MRS total mostró un coeficiente de alfa de Cronbach de 0,86 . Por su parte, la dimensión somática alcanzó alfa de Cronbach de 0,63 (ítem 1, 2, 3 y 11); la psicológica 0,75 (ítem 4, 5, 6, y 7); y la urogenital 0,84 (ítem 8, 9 y 10). Las correlaciones corregidas y el alfa de Cronbach si el ítem se omitiera se presentan en la tabla 1.

Tabla 1. Correlación de cada ítem con la puntuación total corregida (CIPTc) y alfa de Cronbach si el ítem se omitiera (ACIO)

\begin{tabular}{|l|l|l|}
\hline \multicolumn{1}{|c|}{ Ítem } & CIPTc & ACIO \\
\hline 1. Oleadas de calor & 0.721 & 0.839 \\
\hline 2. Taquicardia & 0.450 & 0.856 \\
\hline 3. Problemas de sueño & 0.365 & 0.863 \\
\hline 4. Estado de ánimo depresivo & 0.475 & 0.855 \\
\hline 5. Irritabilidad & 0.653 & 0.845 \\
\hline 6. Ansiedad & 0.520 & 0.852 \\
\hline 7. Cansancio físico y mental & 0.567 & 0.849 \\
\hline 8. Problemas sexuales & 0.761 & 0.833 \\
\hline 9. Problemas de vejiga & 0.508 & 0.856 \\
\hline 10. Sequedad vaginal & 0.661 & 0.841 \\
\hline 11. Problemas musculo/articulares & 0.553 & 0.852 \\
\hline
\end{tabular}


A su vez en la tabla 2 se presentan las comparaciones entre las puntuaciones obtenidas en mujeres posmenopáusicas y premenopáusicas.

Tabla 2. Puntuación de los dominios de la Escala MRS distribuidos de acuerdo con los estados menopaúsicos. Validez nomológica

\begin{tabular}{|c|c|c|c|c|c|}
\hline Dominio & Categoría & Media & $\mathrm{DE}$ & $t^{*}$ & $\mathrm{p}$ \\
\hline Somático & $\begin{array}{l}\text { Posmenopausia } \\
\text { Premenopausia }\end{array}$ & $\begin{array}{l}5,60 \\
3,50\end{array}$ & $\begin{array}{l}2,63 \\
2,29\end{array}$ & 12,69 & 0,001 \\
\hline Psicológico & $\begin{array}{l}\text { Posmenopausia } \\
\text { Premenopausia }\end{array}$ & $\begin{array}{l}3,73 \\
2,61\end{array}$ & $\begin{array}{l}2,58 \\
2,18\end{array}$ & 6,94 & 0,001 \\
\hline Urogenital & $\begin{array}{l}\text { Posmenopausia } \\
\text { Premenopausia }\end{array}$ & $\begin{array}{l}5,15 \\
2,33\end{array}$ & $\begin{array}{l}2,72 \\
2,46\end{array}$ & 16,43 & 0,001 \\
\hline Total & $\begin{array}{l}\text { Posmenopausia } \\
\text { Premenopausia }\end{array}$ & $\begin{array}{c}14,47 \\
8,44\end{array}$ & $\begin{array}{l}6,47 \\
5,94\end{array}$ & 14,53 & 0,001 \\
\hline$* \mathrm{gl}=912$ & & & & & \\
\hline
\end{tabular}

De otro lado, el análisis de factores arrojó que los ítems guardaban factores subyacentes, con coeficiente KMO superior a 0,800 y prueba de Bartlett con valor de $\mathrm{p}$ inferior a 0,05 . Se identificaron dos dimensiones relevantes con valores propios superiores a 1,40. La primera dimensión que dio cuenta del $39,9 \%$ de la varianza y la formaron los ítems $1,7,8,9,10$ y 11 que agrupan principalmente los síntomas tradicionalmente conocidos como somáticos y urogenitales de la menopausia. Y la segunda dimensión explicó el 14,2\% de la varianza y reunió los ítems 2, 3, 4, 5 y 6 que abordan particularmente síntomas emocionales o psicológicos de la menopausia. La correlación de Pearson entre la primera y la segunda dimensión fue alta, 0,432 ( $\mathrm{p}<0.001)$. La primera dimensión tuvo un alfa de Cronbach de 0,86; y la segunda, 0,81 y la varianza total que explicaron las dos dimensiones fue $54,1 \%$ de la varianza total (Tabla 3 ).

Tabla 3. Matriz de coeficientes después de rotación promax $\left({ }^{*}\right)$

\begin{tabular}{|l|c|c|c|}
\hline \multicolumn{1}{|c|}{ Ítem } & Comunalidad & Factor 1 & Factor 2 \\
\hline 1. Oleadas de calor & 0.633 & $\underline{0.791}$ & 0.420 \\
\hline 2. Taquicardia & 0.320 & 0.324 & $\underline{0.558}$ \\
\hline 3. Problemas de sueño & 0.536 & 0.189 & $\underline{0.719}$ \\
\hline 4. Estado de ánimo depresivo & 0.713 & 0.283 & $\underline{0.840}$ \\
\hline 5. Irritabilidad & 0.512 & $\underline{0.582}$ & $\underline{0.637}$ \\
\hline 6. Ansiedad & 0.497 & 0.379 & $\underline{0.700}$ \\
\hline 7. Cansancio físico y mental & 0.358 & $\underline{0.572}$ & 0.406 \\
\hline 8. Problemas sexuales & 0.753 & $\underline{0.867}$ & 0.414 \\
\hline 9. Problemas de vejiga & 0.610 & $\underline{0.748}$ & 0.121 \\
\hline 10. Sequedad vaginal & 0.648 & $\underline{0.803}$ & 0.301 \\
\hline
\end{tabular}


Continuación Tabla 3.

\begin{tabular}{|l|c|c|c|}
\hline \multicolumn{1}{|c|}{ Ítem } & Comunalidad & Factor 1 & Factor 2 \\
\hline 11.Problemas musculo/articulares & 0.370 & $\underline{0.605}$ & 0.312 \\
\hline Valor propio & - & 4.392 & 1.565 \\
\hline Porcentaje de la varianza (\%) & - & 39.9 & 14.2 \\
\hline
\end{tabular}

KMO = 0.882 Prueba de Bartlett, chi cuadrado 4392,55; grados de libertad $=55 ; p=0,001$

* Los coeficientes superiores a 0,550 aparecen subrayados (buen valor).

\section{DISCUSIÓN}

En la presente investigación se observa que la MRS mostró alta consistencia interna, adecuada validez nomológica y una estructura factorial bidimensional en el Climaterio, en un grupo de mujeres indígenas colombianas.

Los resultados de esta investigación son consistentes con trabajos previos que mostraron que la MRS total presenta alta consistencia interna ${ }^{17,18}$. No obstante, la consistencia interna para cada una de las dimensiones tradicionalmente reconocidas fue distinta, dimensión somática presentó consistencia interna inferior a 0,70 mientras que en las dimensiones emocional y urogenital fueron superiores, contrario a lo que observó en investigaciones previas que hallaron mayor consistencia interna para la dimensión somática que para las dimensiones psicológica y urogenital $^{17,18}$.

Se observó que las mujeres en posmenopausia tuvieron puntuaciones significativamente superiores que las mujeres en período de premenopausia en todas las dimensiones y la puntuación total que prueba la validez nomológica de la escala9 ${ }^{9}$ Consistente con los hallazgos de estudios precedentes que observaron un comportamiento similar en las puntuaciones de las escalas en los diferentes períodos del climaterio ${ }^{21-25}$.

En relación con las dimensiones de la MRS, en el presente estudio se identificaron sólo dos grandes dimensiones; la primera con un alto número de ítem para síntomas somáticos y urogenital; y la segunda, una gran participación de ítem psicológicos. Esto es distinto que se presentó en la investigación de introducción de la MRS que identificó claramente tres dimensiones: somática, psicológica y urogenital ${ }^{16}$. No obstante, en un estudio reciente se observó que algunos ítems en los dominios variaron según el origen y contexto de las mujeres participante ${ }^{36}$. Esta observación evidencia que la estructura factorial de la escala se modifica de acuerdo a las características de la población ${ }^{6-8}$.

Es incuestionable que la cultura matiza la expresión a los cambios que se dan a lo largo de la vida. Ya se mencionó que la frecuencia de los síntomas que se atribuyen a la menopausia varía según las distintas características sociales, étnicas y culturales de las participantes en las investigaciones ${ }^{21-32}$. La cultura afecta los estilos de vida, hábitos alimentarios y sexualidad, con la inclusión de las experiencias que se dan en la menopausia, y pueden explicar en parte las diferencias en los síntomas relacionados con la menopausia que se observan en diferentes culturas ${ }^{37-39}$. Por ejemplo, en mujeres indígenas en Australia se observó que los síntomas de menopausia varían según el contexto, urbano o rural (próximo o remoto); además, en algunos grupos, la mujer gana estatus al llegar a la menopausia ${ }^{40}$.

Es necesario tener presente las distorsiones lingüísticas que se introducen involuntariamente en las escalas de medición en los procesos de traducción del idioma original a un segundo idioma o cuando en la traducción media un tercer idioma, como el presente caso que la escala de español se tradujo y adaptó a las lenguas indígenas de las mujeres participantes ${ }^{19,41}$. Y en ocasiones no se encuentran equivalencias exactas entre idiomas, por ejemplo menopausia en inglés (menopause) y el equivalente en japonés $(k o n e n k i)^{42}$.

Este trabajo representa un aporte importante al conocimiento del desempeño de la MRS en una población de mujeres indígenas colombianas y como algunos aspectos sociales o culturales pueden variar el funcionamiento de instrumentos tipo escala de medición. No obstante, presenta la limitación de incluir sólo un grupo limitado de etnias de las que habitan en el país y no se contó con un criterio de referencia para estimar otros indicadores de desempeño de la escala ${ }^{5}$. La 
traducción realizada subjetivamente por la encuestadora y la comprensión de las participantes puede ser factor importante, teniendo en cuenta sobretodo el bajo nivel educativo de las mujeres involucradas. No existe escala valida en las lenguas nativas de las indígenas involucradas. El trabajo aporta visibilización de comunidades indígenas colombianas, que también son elementos importante de estudio en salud en general y en especialmente en el estudio de la calidad de vida en el climaterio. Señala la necesidad de ajustar las herramientas internacionales existentes o la importancia de diseñar herramientas específicas a las condiciones culturales propias de las etnias amerindias colombianas para realizar estudios que presenten puntualmente las alteraciones en la calidad de vida de las mujeres indígenas tanto en premenopausia como en posmenopausia.

Se concluye que la MRS muestra alta consistencia interna, adecuada validez nomológica como ha sido ampliamente señalado en otras comunidades, pero dos dimensiones a diferencia de las identificadas en el estudio inicial en mujeres del considerado mundo desarrollado. Estos resultados muestran la necesidad de corroborar el desempeño de los instrumentos en diferentes poblaciones y probar la escala frente a un criterio de referencia.

\section{DECLARACIÓN SOBRE CONFLICTOS DE INTERESES}

Ninguno que declarar. Financiación parcial para Monterrosa-Castro por medio de la Vicerrectoría de investigaciones de la Universidad de Cartagena. Colombia. Resolución 03707 del 2014, trámite para la obtención de recursos financieros en apoyo al fortalecimiento y sostenibilidad de Grupos de Investigación. Acta de compromiso Número 070-2015 entre Universidad de Cartagena y Alvaro Monterrosa-Castro, como líder del Grupo de Investigación Salud de la Mujer. Agradecimiento a las líderes de las comunidades indígenas que cumplieron labor de encuestadoras para tomar la información.

\section{REFERENCIAS BIBLIOGRÁFICAS}

1. Downing SM, Haladyna TM. Test item development: Validity evidence from quality assurance procedures. Applied Measurement in Education 1997;10(1):61-82.

2. Worthington RD, Whittaker TA. Scale development research. A content analysis and recommendations for best practices. Counseling Psychology 2006; 34(6): 806-838.
3. Roberts P, Priest H, Traynor M. Reliability and validity in research. Nursing Standard 2006;20 (44):41-45.

4. Cook DA, Beckman TJ. Current concepts in validity and reliability for psychometric instruments: Theory and application. American Journal of Medicine 2006;119(2): 166.e7-166.e16.

5. Hulley SB, Cummings SR. Planning the measurement: precision and accuracy. In: Hulley SB, Cummings SR. Designing clinical research. An epidemiologic approach. Baltimore: Williams \& Wilkins; 2001. p. 31-41.

6. Myers K, Winters N. Ten-year review of rating scales. I: Overview of scale functioning, psychometric properties, and selection. J Am Acad Child Adolesc Psychiatry. 2002;41(2):114-22

7. Reise SP, Waller NG, Comrey AL. Factor analysis and scale revision. Psychological Assessment 2000;12(3):287-297.

8. Streiner DL. Figuring out factors: the use and misuse of factor analysis. Can J Psychiatry 1994;39(3):135-140.

9. Adcock R, Collier D. Measurement validity: A shared standard for qualitative and quantitative research. American Political Science Review 2001;95(3):529-546.

10. Cronbach J. Coefficient alpha and the internal structure of test. Psychometrika 1951;16(3): 297-334.

11. Blacker D, Endicott J. Psychometric properties: concepts of reliability and validity. In: Handbook of psychiatric measures. Washington: American Psychiatric Association; 2002. p. 7-14.

12. Pearson K. Determination of the coefficient of correlation. Science 1909; 30(1): 23-25.

13. Spearman C. Correlation calculated from faulty data. British Journal of Psychology 1910; 3(3): 271-295.

14. Shrout PE, Fleiss JL. Intraclass correlations: uses in assessing rater reliability. Psychological Bulletin 1979;86:420-428.

15. Hauser GA, Huber IC, Keller PJ, Lauritzen C, Schneider HPG. Evaluation der klimakterischen beschwerden (menopause rating scale MRS). Zentralblatt für Gynäkologie 1994;116:16-23.

16. Schneider HPG, Hauser GA. The menopause rating scale (MRS II) - clusters of menopausal symptoms. Maturitas 1997;27(Suppl. 1):201-206.

17. Heinemann K, Ruebig A, Potthoff P, Schneider HPG, Heinamann LAJ, Thai DM. The Menopause Rating Scale (MRS) scale: a methodological review. Health and Quality of Life Outcome 2004;2:45-47.

18. Metintas S, Arýkan I, Kalyoncu C, Ozalps J. Menopause Rating Scale as a screening tool in rural Turkey. Rural and Remote Health 2010;10:1-11.

19. Rubio-Stipec M, Hicks MHR, Tsuang MT. Cultural factors influencing the selection, use, and interpretation of psychiatric measures. In: Handbook of psychiatric measures. Washington: American Psychiatric Association; 2002. 
20. Clark MJ. Cross-cultural research: Challenge and competence. International Journal of Nursing Practice 2012; 18 (Suppl. 2):28-37.

21. Chedraui P, Aguirre WC, Hidalgo L, Fayad L. Assessing menopausal symptoms among healthy middle aged women with the Menopause Rating Scale. Maturitas 2007; 57(3): 271-278.

22. Alwi SARS, Awi PYL, Mallik PS, Haizal MNM. The menopausal experience among indigenous women of Sarawak, Malaysia. Climateric 2009;12(6):548-556.

23. López F, Soares DR, d'Andretta AC. Calidad de vida de mujeres en fase de transición menopáusica evaluado por la Menopause Rating Scale (MRS). Revista Chilena de Obstetricia y Ginecología 2010;75(4): 375-382.

24. Chuni N, Chandrashekhar T, Sreeramareddy T. Frequency of symptoms, determinants of severe symptoms, validity of and cut-off score for Menopause Rating Scale (MRS) as a screening tool: A cross-sectional survey among midlife Nepalese women. BMC Women's Health 2011;11:30.

25. Legorreta D, Montaño JA, Hernández I, Salinas C, Hernández-Bueno JA, on the behalf of AMEC Research Committee. Age at menopause, motives for consultation and symptoms reported by 40-59-year-old Mexican women. Climateric 2013;16(4):417-425.

26. Kakkar V, Kaur D, Chopra K, Kaur A, Kaur IP. Assessment of the variation in menopausal symptoms with age, education and working/non-working status in north-Indian sub population using menopause rating scale (MRS). Maturitas 2007;57(3):306-314.

27. Lawton BA, Rose SB, Cormack DM, Stanley J, Dowell AC. The menopause symptom profile of Maori and non-Maori women in New Zealand. Climateric 2008; 11(6): 467-474.

28. Monterrosa A, Blumel JE, Chedraui P, Gomez B, Valdez C. Quality of life impairment among postmenopausal women varies according to race. Gynecological Endocrinology 2009;25(5): 491-497.

29. Rahman SASA, Zainudin ST, Mun VLK. Assessment of menopausal symptoms using modified Menopause Rating Scale (MRS) among middle age women in Kuching, Sarawak, Malaysia. Asian and Pacific Family Medicine 2010;9:5.

30. Krajewska-Ferishah K, Krajewska-Kułak E, Terlikowski S, Wiktor H, Van Damme-Ostapowicz K, Chadzopulu
A, Adraniotis J, Shpakou A. Analysis of quality of life of women in menopause period in Poland, Greece, Belarus and Belgium using MRS Scale. A multicenter study. Advances in Medical Sciences 2010; 52(2):191-195.

31. Krajewska-Ferishah K, Krajewska-Kułak E, Terlikowski S, Wiktor H. Analysis of quality of life women in menopause period in Poland. Prog Health Sci 2011;1(2):52-58.

32. Ojeda E, Monterrosa A, Blümel JE, Escobar-López J, Chedraui P. Severe menopausal symptoms in mid-aged Latin American women can be related to their indigenous ethnic component. Climateric 2011; 14(1):157-163.

33. Kaiser HF. An index of factorial simplicity. Psychometrika 1974; 34(1):31-36.

34. Bartlett MS. Test of significance in factor analysis. British Journal of Psychology 1950; 3(2): 77-85.

35. Gorsuch RL. Exploratory factor analysis: its role in item analysis. Journal of Personality Assessment 1997; 68(3):532-560.

36. Boral S, Borde T, Kentenich H, Wernecke KD, David M. Migration and symptom reporting at menopause: a comparative survey of migrant women from Turkey in Berlin, German women and women in Istanbul. Menopause 2013; 20(2):169-178.

37. Lock M. Menopause: lessons from anthropology. Psychosomatic Medicine 1998; 60(5):410-419.

38. Kilaf E, Kirchengast S. Menopause between nature and culture: menopausal age and climacteric symptoms among Turkish immigrant women in Vienna, Austria. Acta Medica Lituanica 2008;15(1):2-8.

39. Pitkin J. Cultural issues and the menopause. Menopause International 2010;16(2):156-161.

40. Jones EK, Jurgenson JR, Katzenellenbogen JM, Thompson SC. Menopause and the influence of culture: another gap for Indigenous Australian women? BMC Women's Health 2012; 12: 43.

41. Sousa VM, Rojjanasrirat W. Translation, adaptation and validation of instruments or scales for use in crosscultural health care research: a clear and user-friendly guideline. Journal of Evaluation in Clinical Practice 2011;17(2): 268-274.

42. Melby MK, Lock M, Kaufert P. Symptom reporting at menopause: a review of cross-cultural findings. Human Reproduction Update, 2005;11(5):495-512. 